\title{
Dixon MRI
}

National Cancer Institute

\section{Source}

National Cancer Institute. Dixon MRI. NCI Thesaurus. Code C122667.

An imaging method that combines in-phase and out-of-phase echoes to create water-

only and fat-only images. This technique is particularly useful in abdominal imaging at 3T . 